\title{
Prevalência de sobrepeso e obesidade em escolares da região de Parelheiros do município de São Paulo
}

\author{
Prevalence of overweight and obesity in school children of Parelheiros region in São Paulo city, Brazil
}

Anna Luiza N. Fagundes ${ }^{1}$, Denise Carpigiani Ribeiro ${ }^{1}$, Laura Naspitz¹, Luciana Elisa B. Garbelini', Júlia Ketter P. Vieira ${ }^{1}$, Adriana Paulino da Silva ${ }^{1}$, Vitor de Oliveira Lima ${ }^{1}$, Djalma José Fagundes², Patrícia Colombo Compri ${ }^{3}$ Yára Juliano ${ }^{3}$

\section{RESUMO}

Objetivo: Verificar, em uma amostra de escolares de uma região de baixo nível socioeconômico, a prevalência de sobrepeso e obesidade.

Métodos: Estudo transversal com uma amostra de 218 crianças selecionadas de modo aleatório, a partir de um universo estimado de 1.500 crianças e adolescentes, com idade entre seis e 14 anos, provenientes de três escolas de ensino fundamental de Parelheiros, São Paulo, sem distinção de sexo ou etnia. Foram excluídas aquelas portadoras de qualquer doença metabólica ou endócrina diagnosticada ou em tratamento. Os indivíduos foram submetidos à avaliação antropométrica, com medida do peso corporal e altura para cálculo do índice de massa corpórea e relação peso/altura. Todas as crianças responderam a um questionário aplicado pelos pesquisadores sobre hábitos alimentares.

Resultados: A prevalência de obesos e de portadores de sobrepeso foi respectivamente 14,7 e 16,5\%. Entre os alunos obesos e com sobrepeso, o baixo consumo de frutas (10,8 e $10,8 \%)$, de verduras e legumes $(16,4$ e $9,1 \%)$ e o alto consumo de doces $(72,2$ e 78,1\%) foram associados ao excesso de peso. A prática esportiva esteve ausente ou escassa nos obesos $(81,3 \%)$ e portadores de sobrepeso $(77,8 \%)$.

Conclusões: Os alunos avaliados, embora provenientes de uma região pobre de São Paulo, apresentaram um perfil de transição nutricional, com altas taxas de obesidade e sobrepeso.

Palavras-chave: obesidade; sobrepeso; criança; adolescente; transtornos da nutrição infantil.

\section{ABSTRACT}

Objective: To establish the prevalence of overweight and obesity in school children from low socioeconomical status.

Methods: This cross sectional study randomly enrolled 218 eighteen students out of 1.500 students with six to 14 years old from three schools located in Parelheiros, São Paulo, Brazil. Children with a diagnosis and or treatment of metabolic or endocrine diseases were excluded. The studied children had an anthropometric evaluation of weight and height, and body mass index was calculated. All children answered a questionnaire about daily food consumption and physical activity.

Results: The prevalence of obesity and overweight were respectively 14.7 and $16.5 \%$. Among these students, the low consumption of fruits (10.8 and $10.8 \%)$, vegetables (16.3 and $9.1 \%)$ and the high consumption of candies (72.2 and $78.1 \%$ ) were associated with weight excess. Poor physical activities was common among obese $(81.3 \%)$ and overweight (77.8\%) students.

Conclusions: The evaluated students, despite living in a poor region of São Paulo, showed a profile of nutritional transition, with high obesity and overweight rates.

Key-words: obesity; overweight; child; adolescent; child nutrition disorders.
${ }^{1}$ Acadêmica de Medicina da Universidade de Santo Amaro (Unisa), São Paulo, SP, Brasil

${ }^{2}$ Professor associado e livre-docente do Departamento de Cirurgia da Universidade Federal de São Paulo, São Paulo, SP, Brasil

${ }^{3}$ Professora titular da Disciplina de Saúde Coletiva da Faculdade de Medicina da Unisa, São Paulo, SP, Brasil
Endereço para correspondência:

Anna Luiza N. Fagundes

Rua Henrique Peres, 91

CEP 03123-070 - São Paulo/SP

E-mail: zulinha@hotmail.com

Recebido em: 19/10/2007

Aprovado em: 29/4/2008 


\section{Introdução}

A obesidade é uma doença crônica caracterizada pelo acúmulo excessivo de energia, sob a forma de triglicérides, no tecido adiposo distribuído pelo corpo e pode provocar prejuízos à saúde, por facilitar o desenvolvimento ou agravamento de doenças associadas. Já o sobrepeso é o excesso de peso previsto para o sexo, altura e idade, de acordo com os padrões populacionais de crescimento, podendo representar ou não excesso de gordura corporal ${ }^{(1-3)}$.

Atualmente, o aumento da prevalência de sobrepeso em graus variados na população infantil é observado em diversas partes do mundo e tem repercussões sobre a situação da saúde e qualidade de vida das crianças. No Brasil, nota-se a presença de transição nutricional, caracterizada pela redução na prevalência dos deficits nutricionais e ocorrência mais expressiva de sobrepeso e obesidade não só na população adulta, mas também em crianças e adolescentes. Dados da Pesquisa Nacional sobre Saúde e Nutrição indicam a presença de cerca de 1,5 milhão de crianças obesas, com maior prevalência em meninas, sendo, nas regiões Sul e Sudeste, sua ocorrência o dobro da região Nordeste. Há maior de prevalência de obesidade nas faixas de renda intermediária e $\operatorname{alta}^{(4,5)}$.

A obesidade é uma doença de difícil controle, com alto percentual de insucesso terapêutico e de recidivas, podendo apresentar, na sua evolução, sérias repercussões orgânicas e psicossocias. Os pilares fundamentais no tratamento da obesidade são as modificações de comportamento e de hábitos de vida, que incluem mudanças no plano alimentar e na atividade física $^{(3)}$. Crianças obesas têm maior probabilidade de se tornarem adultos obesos. Segundo Gamba e Barros Júnior $^{(3)}$, o controle da obesidade em adultos tem se mostrado pouco eficaz, parecendo mais adequado identificar as crianças de risco e procurar fazer a profilaxia do distúrbio nutricional. O diagnóstico precoce e as intervenções no período crítico do desenvolvimento da obesidade (infância e adolescência) têm sido recomendados para evitar desfechos desfavoráveis na idade adulta ${ }^{(4)}$.

Deste modo, ao considerar a área geográfica atendida pelo Departamento de Saúde Coletiva da Universidade de Santo Amaro (região sul do município de São Paulo, subdistrito de Parelheiros, com população de baixo nível socioeconômico $)^{(5)}$, percebeu-se a necessidade de buscar informações a respeito de hábitos alimentares e atividade física de crianças e adolescentes residentes no município, a fim de elaborar programas de intervenção específicos.
Neste contexto, o objetivo desta pesquisa foi verificar, em uma amostra de escolares, a prevalência de sobrepeso e obesidade, procurando estabelecer as relações com hábitos alimentares e atividade física.

\section{Métodos}

A pesquisa foi submetida à apreciação e aprovação do Comitê de Ética em Pesquisa do Hospital Geral do Grajaú. Todos os pais ou responsáveis legais das crianças e adolescentes assinaram o termo de consentimento livre e esclarecido.

Realizou-se um estudo transversal com uma amostra de 218 crianças e adolescentes de seis a 14 anos, de um total de 1.500 alunos nesta faixa etária, matriculados nas três escolas (14,5\%), sem distinção de sexo, etnia ou período de frequiência às aulas (matutino/vespertino). Os alunos foram escolhidos de modo aleatório em três escolas municipais de ensino fundamental (Escola Municipal de Ensino Fundamental Manuel Vieira de Queiroz Filho, Escola Municipal de Ensino Fundamental Pedro Geraldo Shunk e Escola Municipal de Ensino Fundamental Vargem Grande), de um total de cinco unidades educacionais da Coordenadoria de Educação de Parelheiros, região sul de São Paulo. Foram excluídos os sujeitos com doença metabólica diagnosticada ou em tratamento, aqueles que faziam uso de qualquer medicação de uso contínuo ou que tiveram algum tipo de tratamento médico nos últimos trinta dias, segundo informação fornecida pelos responsáveis pela criança junto à escola.

O estudo foi realizado por acadêmicos de medicina da universidade, em parceria com a Secretaria Municipal de Saúde e Educação e sob supervisão do Corpo Docente da Disciplina de Saúde Coletiva, durante o mês de outubro de 2006.

Todos os alunos já tinham o consentimento dos pais ou responsáveis exigido pela escola para realização da pesquisa. Os alunos responderam a um único questionário semiestruturado com doze questões de múltipla escolha. Tal instrumento foi construído e aplicado pelos pesquisadores, com questões que avaliavam os hábitos alimentares, a realização de refeições, os horários e a freqüência da utilização na dieta de frutas, verduras e doces, além da freqüência e do local da prática esportiva e antecedente familiar quanto à obesidade. As respostas referentes ao hábito alimentar e à prática esportiva podiam ser sim ou não e, em caso de resposta positiva, questões referentes à freqüência eram aplicadas (opções: uma ou duas vezes por semana, três ou quatro vezes por semana, cinco ou seis vezes por semana ou todos os dias). 
Quanto aos antecedentes familiares, constavam apenas as opções sim ou não.

A seguir, os escolares foram submetidos à avaliação antropométrica pela medida do peso corporal $(\mathrm{kg})$ e altura (cm) em balança plataforma Filizola ${ }^{\circledR}$ com capacidade de $150 \mathrm{~kg}$, precisão de $100 \mathrm{~g}$ e antropômetro já acoplado, estando os participantes sem sapatos e trajando roupas leves. A partir dos dados coletados, foi calculada a relação peso/ estatura (P/E) usando como referência as curvas do National Center for Health Statistics ${ }^{(6)}$. Definiu-se como obesidade valor $>120 \%$; sobrepeso como $<120 \%$ e $>110 \%$; eutrofia como $<110 \%$ e $>95 \%$; e desnutrição $<95 \%{ }^{(3,7)}$.

Os dados foram analisados no programa Epi-Info ${ }^{\circledR}$ v.6.04. Para relacionar o estado nutricional com o gênero do aluno, o hábito alimentar, a prática esportiva e os antecedentes familiares, aplicou-se o teste do qui-quadrado. Para rejeição da hipótese de nulidade considerou-se o nível de significância $p \leq 0,05^{(8)}$.

\section{Resultados}

Dos 218 alunos estudados, 103 eram do sexo masculino e 115 do feminino. A idade mínima foi de seis e máxima de 14 anos, com média de 10,2 e mediana de dez anos (Tabela 1). A Tabela 2 mostra a distribuição dos alunos segundo as categorias nutricionais (eutrofia, sobrepeso, obesidade e desnutrição), de acordo com a classificação da relação peso/estatura.

Já a Tabela 3 mostra a freqüência, em porcentagem, da ocorrência dos hábitos alimentares diários, sendo os mais relevantes o número de refeições ao dia, alimentar-se entre

Tabela 1 - Distribuição da população estudada, segundo a idade

\begin{tabular}{lc}
\hline Idade (anos) & Número de entrevistados \\
\hline 6 & 10 \\
7 & 41 \\
8 & 20 \\
9 & 28 \\
10 & 28 \\
11 & 14 \\
12 & 6 \\
13 & 41 \\
14 & 30 \\
\hline Total & 218 \\
\hline
\end{tabular}

as principais refeições, o consumo de doces, legumes e verduras. Observa-se uma diferença significante entre obesos, portadores de sobrepeso e eutróficos no que concerne a comer merenda escolar, jantar, comer entre as refeições e comer verduras e legumes.

Quanto aos diversos hábitos nutricionais e de atividades físicas pesquisados, observou-se que os portadores de sobrepeso e os obesos são os que menos praticavam esporte durante a semana. Dos que responderam que a freqüência da prática esportiva era de duas a três vezes por semana, $18,2 \%$ eram obesos e 16,9\% tinham sobrepeso, comparados a $41,6 \%$ dos eutróficos. Da mesma forma, os portadores de sobrepeso e os obesos consumiam menos verduras e legumes, sendo que, daqueles que faziam uso desses alimentos diariamente, apenas 16,4\% tinham sobrepeso e $9,1 \%$ eram obesos, em comparação a $42,3 \%$ dos eutróficos. Os portadores de sobrepeso e obesidade também foram os que menos relataram o consumo de frutas: daqueles que as consumiam três a quatro vezes por semana, $10,8 \%$ tinham sobrepeso e $10,8 \%$ eram obesos, comparados a $43,2 \%$ dos eutróficos.

\section{Discussão}

O diagnóstico da obesidade pode ser feito por diversos métodos, porém os antropométricos são os de mais fácil execução, não invasivos, de baixo custo e os mais adequados para a prática diária ${ }^{(3)}$. Considerando a faixa etária em estudo, optou-se pela escolha da relação peso/estatura por ser a mais fidedigna aos propósitos da pesquisa, uma vez que a principal restrição ao uso do índice de massa corpórea (IMC) é a sua especificidade em relação a sexo, idade, maturação sexual e etnia, exigindo uma relativização dos dados encontrados e diferentes padrões de referência ${ }^{(2)}$. Além disso, o IMC não reflete as grandes mudanças na composição corporal que ocorrem na adolescência, com maior acúmulo de gordura nas meninas e de massa muscular nos meninos. Sendo assim, meninos e

Tabela 2 - Distribuição da população estudada, segundo o índice peso/estatura (P/E)

\begin{tabular}{lcc}
\hline Estado nutricional & Número & $\%$ \\
\hline Eutrofia & 94 & 43,1 \\
Sobrepeso & 36 & 16,5 \\
Obesidade & 32 & 14,7 \\
Desnutrição & 56 & 25,7 \\
\hline Total & 218 & 100 \\
\hline
\end{tabular}


Tabela 3 - Distribuição da freqüência (\%) de gênero, hábitos alimentares e comportamentais, prática esportiva e antecedentes familiares na população estudada, segundo as categorias nutricionais: eutrofia, sobrepeso, obesidade e desnutrição

\begin{tabular}{|c|c|c|c|c|c|}
\hline & Eutrofia & Sobrepeso & Obesidade & Desnutrição & $p$ \\
\hline Gênero masculino & 44,7 & 47,2 & 43,8 & 53,6 & NS \\
\hline \multicolumn{6}{|l|}{ Hábitos alimentares presentes } \\
\hline Café da manhã & 76,6 & 69,5 & 75,0 & 80,3 & NS \\
\hline Almoço & 88,3 & 91,7 & 93,8 & 91,0 & NS \\
\hline Merenda & 64,9 & 41,7 & 71,9 & 58,9 & $<0,05$ \\
\hline Jantar & 89,3 & 77,8 & 84,4 & 96,4 & $<0,05$ \\
\hline Comer entre refeições & 70,2 & 58,3 & 75,0 & 44,7 & $<0,01$ \\
\hline Horário para refeições & 45,7 & 52,8 & 28,1 & 46,4 & NS \\
\hline Consumo diário de doces & 57,0 & 72,2 & 78,1 & 51,8 & $<0,05$ \\
\hline Consumo diário de doces & 63,2 & 53,9 & 59,0 & 42,9 & NS \\
\hline Consumo de frutas & 93,6 & 91,7 & 93,8 & 91,0 & NS \\
\hline Consumo de verduras/legumes & 83,0 & 72,2 & 59,4 & 85,7 & $<0,02$ \\
\hline \multicolumn{6}{|l|}{ Prática esportiva } \\
\hline Refere presente & 79,8 & 77,8 & 81,3 & 89,3 & NS \\
\hline Refere na escola & 54,6 & 46,4 & 38,5 & 48,0 & NS \\
\hline Obesidade na família & 53,2 & 50,0 & 59,4 & 53,6 & NS \\
\hline Dorme após refeições & 29,8 & 8,3 & 34,5 & 25,0 & NS \\
\hline
\end{tabular}

NS: não significante

meninas, de diferentes idades, com variadas quantidades de gordura corporal, podem ter valores semelhantes de IMC, fato que justifica a discrepância observada entre os métodos antropométricos utilizados e a opção pela utilização do peso/ estatura na análise dos dados ${ }^{(2,4)}$

Várias pesquisas enfatizam o aumento de peso infantil nos últimos anos. Dados com relação à obesidade infantil são ainda escassos, uma vez que este problema era freqüentemente associado a distúrbios genéticos ou a conseqüências de graves lesões cerebrais ocorridas durante o nascimento. Até mesmo na época em que a International Obesity Task Force se reuniu pela primeira vez para diagnosticar a epidemiologia e outros aspectos da saúde pública da doença, havia pouca informação sobre a obesidade infantil no mundo e nenhum sistema amplamente aceito para relatá-la ${ }^{(9)}$. No Brasil, a rápida diminuição das taxas de desnutrição associada ao aumento nas taxas de obesidade têm ocorrido em curto intervalo de tempo, despertando preocupação e interesse de pediatras ${ }^{(10)}$. Novas políticas públicas em relação aos cuidados alimentares e nutricionais com as crianças devem ser elaboradas.

Estudos anteriores, com alunos de dez a 13 anos provenientes de 11 escolas da rede pública de São Paulo, mostraram índices de 10,3 e de 11,4\% para obesidade e para o sobrepeso, respectivamente ${ }^{(11,12)}$. Na região de Parelheiros, considerada uma das mais pobres do município de São Paulo e onde há baixo nível de escolaridade, os achados foram coerentes com a literatura, pois se encontrou prevalência de obesidade de $14,7 \%$ e de sobrepeso de $16,5 \%$, em crianças de seis a 14 anos. No entanto, em comparação a outros estudos, notou-se alguma discrepância nos resultados observados.

A maior prevalência de obesidade no presente trabalho $(14,6 \%)$, comparada à de crianças e de adolescentes toda a região Sudeste $(9,8 \%)$ pode ser atribuída à expansão da região analisada. Essa expansão aumenta a chance de mudanças no estilo de vida, no padrão alimentar (hábitos alimentares mais adequados e variados, menos acesso a alimentos industrializados), mudanças também no meio ambiente, nos fatores culturais, econômicos e sociais, no padrão de atividade física (até mesmo por causa do trabalho rural), na estrutura familiar, com conflitos psicoafetivos. Tais mudanças geram aumento na porcentagem de obesidade $\mathrm{s}^{(3,6,12-14)}$.

Analisando os resultados do estudo, verificou-se que ser do gênero feminino ou do masculino não foi um fator relevante para o sobrepeso nem para a obesidade. A faixa etária média abordada foi de 10,2 anos, na qual não há produção de gran- 
des quantidades de hormônios sexuais, que têm influência na produção e distribuição de gordura corporal ${ }^{(2)}$.

Sabe-se que a merenda escolar é extremamente importante para atender os requisitos nutricionais de crianças em fase escolar, porém, neste estudo, a maioria das crianças e adolescentes entrevistados tinha o hábito de almoçar e de ingerir o café da manhã, independentemente do estado nutricional. Contudo, muitas delas também consumiam a merenda escolar (sendo considerada como outro almoço, devido aos seus componentes), por isso, neste caso, a merenda poderia se associar à obesidade ${ }^{(15)}$. Observa-se ainda que os portadores de sobrepeso e os obesos são aqueles que menos jantam. A literatura mostra que omitir refeições é extremamente prejudicial a uma dieta ideal, contribuindo para o aumento do risco de obesidade em crianças e em adolescentes ${ }^{(1)}$. Além disso, nas crianças com obesidade verifica-se o hábito de comer entre as refeições, reduzindo o apetite e interferindo no jantar.

As transformações provocadas pelo estilo de vida moderno levam ao consumo excessivo de produtos gordurosos, doces e bebidas açucaradas, aumentando as chances das crianças e adolescentes desenvolverem prejuízos à auto-imagem, isolamento social e problemas educacionais e econômicos ${ }^{(1,3,5)}$.

A manutenção estável do peso e da composição corporal resulta de um balanço preciso entre a ingestão e o gasto energético, sendo que um desequilíbrio nesta relação desencadeia o processo da obesidade. Os doces são alimentos altamente calóricos e devem estar presentes em quantidades ponderadas em uma dieta equilibrada para não alterar tal balanço ${ }^{(13)}$. Observou-se haver diferença significante no consumo de doces diário na casuística avaliada: portadores de sobrepeso e obesos consomem quantidades maiores de doces em relação às crianças dos demais estados nutricionais.

É responsabilidade dos pais oferecerem uma variedade de alimentos adequados às crianças e diminuir a disponibilidade de alimentos excessivamente calóricos ${ }^{(3)}$. As frutas são consideradas importantes fontes de vitaminas e fibras e recomendadas para a constituição de uma dieta equilibrada. Todavia, sua ingestão ou sua falta na alimentação não determinaram o desenvolvimento do quadro de obesidade ou de sobrepeso nas crianças e adolescentes pesquisados. Portanto, o consumo de frutas, independente do estado nutricional, não foi considerado fator relevante para o excesso de peso.

Não somente o volume alimentar tem sido relacionado à obesidade, como também a composição da dieta pode exercer influência no desenvolvimento da doença. As crianças e os adolescentes obesos, seguidos pelos que apresentam sobrepeso, são aqueles que menos ingeriram verduras e legumes.
O consumo insuficiente de hortaliças aumenta o risco de doenças crônicas não transmissíveis, como as cardiovasculares e alguns tipos de câncer. Tais doenças estão entre os dez fatores de risco que mais causam mortes e doenças em todo o mundo. Estima-se que o consumo de hortaliças no Brasil corresponda a menos da metade das recomendações nutricionais, sendo ainda mais deficiente em famílias de baixa renda ${ }^{(16,17)}$. Estes alimentos, apesar de essenciais, normalmente não são de agrado das crianças e tendem a estar presentes em quantidades insuficientes e a serem substituídos por outros menos adequados. De acordo com a Tabela 3, o hábito de não consumir verduras e legumes esteve associado a maior freqüência de sobrepeso e obesidade ${ }^{(1,5)}$.

A atividade física é importante para o desenvolvimento de crianças saudáveis, ajudando a queima de calorias e evitando o excesso de peso ${ }^{(13)}$. A menor ocorrência da prática esportiva na escola ocorreu nos obesos e naqueles com sobrepeso. Quando estas crianças eram praticantes de esportes, o faziam com menor frequiência semanal do que os eutróficos. Ressalta-se que nenhuma questão relacionada à prática esportiva se mostrou como fator relevante neste estudo, uma vez que as aulas de Educação Física eram obrigatórias a todos os alunos e, dessa maneira, a maioria dos alunos entrevistados respondeu praticar esporte no mínimo uma vez por semana.

Os pilares fundamentais do tratamento da obesidade são as modificações de comportamento e de hábitos de vida que incluem, primeira e principalmente, mudanças no plano alimentar, seguidas de incentivo à atividade física. Sabe-se que isto deve ser feito com cuidado, uma vez que tanto a prática esportiva como o ajuste da dieta precisam ser acompanhados por profissionais especializados ${ }^{(3)}$. No tratamento da criança obesa não há espaço para dietas restritivas, já que os níveis de vitaminas e micronutrientes ficam demasiadamente reduzidos, levando a prejuízos para o crescimento e desenvolvimento. É importante que as mudanças de comportamento propostas para crianças e adolescentes obesos sejam estruturadas adequadamente para evitar distúrbios alimentares posteriores, como os encontrados em adultos que apresentam dificuldade em reduzir o peso corporal ${ }^{(3,14,18)}$.

Os pais precisam controlar os tipos de alimentos oferecidos e os horários das refeições. A criança deve ter liberdade de escolher a quantidade dos alimentos e quais dos alimentos irá comer. A dinâmica familiar assume papel considerável na mudança de práticas alimentares para controle ou tratamento da obesidade. Uma criança com pais obesos tem $80 \%$ de chance de apresentar o mesmo perfil e esse risco cai pela metade se só um dos pais é obeso. Se nenhum pai apresentar obesidade, a 
criança tem risco de $7 \%$ de se tornar obesa ${ }^{(3,14,19)}$. Neste estudo, apesar de não apresentar significância estatística, mais da metade dos obesos possuía algum familiar com sobrepeso ou obesidade. Quanto aos eutróficos, aproximadamente um terço possuía um familiar com sobrepeso ou obesidade (Tabela 3).

Os dados aqui mostrados permitiram verificar que, embora provenientes de uma região pobre, os alunos do ensino fundamental com idade entre seis e 14 anos apresentaram um perfil de transição nutricional, com prevalência de $31,2 \%$ de sobrepeso e obesidade. Os dados também sugerem que o consumo de merenda oferecida pela escola, entre os intervalos

\section{Referências}

1. Lima SC, Arrais RF, Pedrosa LFC. Avaliação da dieta habitual de crianças e adolescentes com sobrepeso e obesidade. Rev Nutr 2004;17:469-77.

2. Sigulem DM, Taddei JAAC, Escrivão MAMS, Devincenzi MU. Obesidade na infância e na adolescência. Compacta Nutr 2001;2:7-15.

3. Gamba EM, Barros Filho AA. A utilização do índice de massa corporal na avaliação da obesidade na infância: vantagens e limitações. Rev Paul Pediatr 1999;17:181-9.

4. Triches RM, Giugliani ER. Obesidade, práticas alimentares e conhecimentos de nutrição em escolares. Rev Saude Publica 2005;39:541-7.

5. Brasil - Ministério da Saúde - DATASUS [homepage on the Internet]. Informações de Saúde - Estatísticas Vitais - Mortalidade e Nascidos Vivos: nascidos vivos desde 1994 [cited 2007 Feb 10]. Available from: http://tabnet.datasus. gov.br/cgi/deftohtm.exe?sinasc/cnv/nvuf.def

6. Estados Unidos - Department of Health and Human Services - NCHS [homepage on the Internet]. Centers for Disease Control and Prevention - Growth Charts: Selected Z-scores Values. [cited 2008 Mar 07]. Available from: http:// www.cdc.gov/nchs/about/major/nhanes/growthcharts/zscore/zscore.htm

7. Moretti K, Beyruti M, Esperança LM, Monegaglia AP, Oliva AB, Brandão FA et al. Prevalência de risco de sobrepeso em escolares de 10 a 13 anos na cidade de São Paulo. Rev Bras Nutr Clin 2000;15:261-6.

8. Veiga GV, Vieira ACR, Alvarez MM, Pereira RC. Índice de massa corporal na avaliação de sobrepeso e obesidade em crianças e adolescentes: concordâncias e controvérsias. Nutrire Rev Soc Bras Aliment Nutr 2004;28:109-24.

9. James WP. Tendências globais da obesidade infantil - conseqüências a longo prazo. Anais Nestlé 2002;62:1-11.

10. Fernandes IT, Gallo PR, Advíncula AO. Avaliação antropométrica de préescolares do município de Mogi-Guaçú, São Paulo: subsídio para políticas das grandes refeições, o consumo de doces, o baixo consumo de frutas e verduras e a ausência ou escassez de atividades físicas regulares foram fatores associados a estes estados nutricionais. A interpretação destes dados será importante para traçar novos programas de saúde coletiva para a comunidade estudada.

\section{Agradecimentos}

À professora Maria do Carmo Negrini Fagundes pela correção ortográfica da língua inglesa. públicas de saúde. Rev Bras Saude Mater Infant 2006;6:217-22.

11. Monteiro P, Victora C, Barros F. Fatores de risco sociais, familiares e comportamentais para obesidade em adolescentes. Rev Panam Salud Publica 2004;16:250-8.

12. Sotelo YOM, Colugnati FAB, Taddei JAA. Prevalência de sobrepeso e obesidade entre escolares da rede pública segundo três critérios de diagnóstico antropométrico. Cad Saude Publica 2004;20:233-40.

13. Sabia RV, Santos JE, Ribeiro RPP. Efeito da atividade física associada à orientação alimentar em adolescentes obesos: comparação entre o exercício aeróbio e anaeróbio. Rev Bras Med Esporte 2004;10:349-55.

14. Levy-Costa RB, Sichieri R, Pontes NS, Monteiro CA. Disponibilidade domiciliar de alimentos no Brasil: distribuição e evolução (1974-2003). Rev Saude Publica 2005;39:530-40.

15. Brasil - Ministério da Educação - MEC [homepage on the Internet]. Fundo Nacional de Desenvolvimento da Educação (FNDE) - Alimentação Escolar [cited 2008 Mar 07]. Available from: http://www.fnde.gov.br/home/index. jsp?arquivo=alimentacao_escolar.html

16. Giugliano R, Carneiro EC. Fatores associados à obesidade em escolares. J Pediatr (Rio J) 2004;80:17-22.

17. Rodrigues EM, Boog MC. Problematização como estratégia de educação nutricional com adolescentes obesos. Cad Saude Publica 2006;22: 923-31.

18. World Health Organization. The world health report 2002 - reducing risks, promoting healthy life. Geneva: WHO; 2002.

19. Novaes JF, Franceschini SCC, Priore SE. Hábitos alimentares de crianças eutróficas e com sobrepeso em Viçosa, Minas Gerais, Brasil. Rev Nutr 2007;20:633-42 